\title{
LIGAND FREE Pd CATALYZED CYCLIZATION-INFLUENCE OF STERIC HINDRANCE
}

\author{
MUHAMMAD NADEEM ARSHAD ${ }^{a, b, c *}$, WASEEQ AHMAD SIDDIQUI* , \\ ISLAM ULLAH KHAN ${ }^{c}$ AND ABDULLAH M. ASIRI ${ }^{a, b}$ \\ ${ }^{a}$ Chemistry Departament Faculty of Science, King Abdulaziz University Jeddah 21589, Saudi Arabia. \\ ${ }^{b}$ Centre of Excellence for Advanced Materials Research (CEAMR) King Abdulaziz University Jeddah 21589, Saudi Arabia. \\ ${ }^{c}$ Materials Chemistry Laboratory, Department of Chemistry, GC University, Lahore-54000, Pakistan. \\ ${ }^{d}$ Department of Chemistry, University of Sargodha, Sargodha-40100, Pakistan.
}

\begin{abstract}
Synthesis of sultams was performed using ligand free palladium catalyst following Heck cyclization. The diverse synthesized intermediates in this strategy includes halogenated sulfonyl chloride, alkyl/aryl sulfonamides, $N$-alkylation using halogenated alkenyl reagents followed by improved Heck cyclization with $\mathrm{Pd}(\mathrm{OAc})_{2} / \mathrm{DIPA}$ in 1-methyl-pyrolidin-2-one/toluene. In this way, isomeric six membered sultams with endocyclic \& exocyclic double bonds (benzothiazine) and seven membered (thiazipene) were obtained. Endocyclic double bond isomer was dominates in respect of yield over the exocyclic double bond isomer. The isolated amounts of endocyclic sultams and thiazipene were almost equal in amounts. The intermediates and final products were characterized using IR, ${ }^{1} \mathrm{H}-\mathrm{NMR}$, ${ }^{13} \mathrm{C}-\mathrm{NMR}$ and EI-MS techniques.
\end{abstract}

Key words; cyclic sultams, 1,2-benzothiazine, ligand free Heck reaction

\section{INTRODUCTION}

Synthesis of new organic compounds is a highly cherished sector of chemistry. After the first Kolbe's synthesis developed for construction of the carbon-carbon (C-C) bond, literature provides references like, Grignard-, aldol- and pericyclic type of reactions. Formation of $\mathrm{C}-\mathrm{C}$ bond through cleavage of $\mathrm{C}-\mathrm{H}$ bond via metal-catalyzed reaction has been developed as the most attractive research area for organic, as well as, organometallic chemists in synthesis of homo/heterocyclic compounds ${ }^{1-4}$. The inestimable significance of these transition metal-catalyzed synthetic transformations has been disclosed by Stille ${ }^{5}$, Negishi ${ }^{6}$, Suzuki-Miyaura ${ }^{7}$, Hiyama ${ }^{8}$ and palladiumcatalyzed Heck cross-couplings [9] using organohalides and other surrogates in synthetic chemistry ${ }^{10,11}$. The traditional Heck reaction drives through: i) a suitable amount of homogenous/heterogeneous palladium (0/II) catalyst, ii) a ligand, usually phosphine and iii) a suitable base. These catalyst systems afford different drawbacks including air/moisture sensitivity and extra $\operatorname{cost}^{12}$. Therefore, there is always room for developing new catalyst systems which will be more advantageous with respect to operation and cost.

Among heterocycles, the benzothiazines ${ }^{13}$ are important molecules to serve as anti-cancer ${ }^{14-16}$, analgesic ${ }^{17}$, anti-microbial ${ }^{18}$, anti-vira ${ }^{19}$, antiinflammatory ${ }^{20-22}$, anti-pyretic ${ }^{23}$, anti-diabetic ${ }^{24}$, anti-hypertensive ${ }^{25}$, antioxidant $^{26}$, herbicidal ${ }^{27}$, and fungicidal ${ }^{28}$, agents. On the other hand, these are reported to exhibit the high vaso-relaxant potency ${ }^{29}$ as Potassium Channel Openers (KCOs). The rapid demand for the synthesis of libraries of these types of medicinal compounds with high throughput activities led us towards the synthesis of sultams. Since the time Braun et al synthesized first benzothiazine, thousands of its derivatives have been synthesized and checked for their biological activities ${ }^{30-33}$. Initially, the most famous and longtime applied methodology for the synthesis of benzothiazine and thiazepine derivatives involved Gabriel-Colman rearrangement ${ }^{34}$. The most recent attempts applied for preparation of these heterocyclic molecules are now using palladium as catalys $\mathrm{s}^{35,36}$ accompanied with a bulky neutral ligand such as triphenylphosphine.

The aforementioned literature together with our already existing program to synthesize new non-steroidal anti-inflammatory drugs (NSAIDs) ${ }^{37-41}$ and their crystallographic studies ${ }^{42,43}$, cultivated our interest to develop a new methodology. The present communication is an outcome of this effort whereby we are reporting a convenient, straightforward and ligand-free palladium catalyzed arylation targeted towards synthesis of six-membered sultams only.

\section{EXPERIMENTAL}

The reactions sensitive to air/moisture were carried out under appropriate conditions. The raw materials were purchased from Merck, BDH, Alfa Aesar or Fluka and used without purification. Standard methods were applied to purify all the solvents and reagents, where applicable. Merck manufactured TLC plates were used to monitor the progress of reactions. ${ }^{1} \mathrm{H}-\mathrm{NMR}$ and
${ }^{13} \mathrm{C}-\mathrm{NMR}$ spectra were recorded on a varian AM 400 instrument. Chemical shifts are reported in ppm referenced to the residual solvent signal. IR spectra were recorded on a Perkin Elmer 1600-FT spectrometer. Melting points were recorded on Electrothermal (Griffin 1090) melting point apparatus and are reported as uncorrected.

Synthesis of 2-bromo- $N$-methyl(or $\quad N$-2,3-dimethyl-phenyl,)benzenesulfonamide $(4,5)$

$\mathrm{Et}_{3} \mathrm{~N}(0.23 \mathrm{~g}, 2.29 \mathrm{mmol})$ was added to the solution of 2-bromobenzenesulfonyl chloride ${ }^{44}(0.25 \mathrm{~g}, 1 \mathrm{mmol})$ and methylamine hydrochloride (or $\mathrm{N}$-2,3-dimethylphenyl) $(1.35 \mathrm{mmol})$ in dichloromethane (DCM) under ice, drop wise. After the completion of addition the mixture was stirred at room temperature for $10 \mathrm{hrs}$ and quenched with saturated $\mathrm{NH}_{4} \mathrm{Cl}$ solution. The organic layer was separated and aqueous layer was extracted with AcOEt. The combined organic layer was evaporated under vacuum after drying over anhydrous $\mathrm{Na}_{2} \mathrm{SO}_{4}$ to obtained yellow oil

2-Bromo- $N$-methylbenzenesulfonamide (4)

Yield; $96.89 \%$, IR (neat) $v_{\max }: 3319,1314,1160 \mathrm{~cm}^{-1},{ }^{1} \mathrm{H}-\mathrm{NMR}:(400$ $\mathrm{MHz})\left(\mathrm{CDCl}_{3}\right) \delta: 8.18(1 \mathrm{H}, \mathrm{d}$, aromatic $), 7.78(1 \mathrm{H}, \mathrm{d}$, aromatic $), 7.42(2 \mathrm{H}, \mathrm{m}$, aromatic), $5.15(1 \mathrm{H}, \mathrm{s}, \mathrm{NH}), 2.58\left(3 \mathrm{H}, \mathrm{d}, \mathrm{CH}_{3}\right),{ }^{13} \mathrm{C}-\mathrm{NMR}$ : (400 MHz), $\left(\mathrm{CDCl}_{3}\right)$ $\delta: 142.65,140.40,138.28,137.85,133.78,126.24,29.72$,

2-Bromo- $\mathrm{N}$-(2,3-dimethyl-phenyl)-benzenesulfonamide (5)

Light brown solid, Yield: $74.4 \%$, MP: $131-132{ }^{\circ} \mathrm{C}$, IR (KBr) $v_{\max }: 3319$, 1314, $1160 \mathrm{~cm}^{-1},{ }^{1} \mathrm{H}-\mathrm{NMR}:(400 \mathrm{MHz})\left(\mathrm{CDCl}_{3}\right) \delta: 7.92(1 \mathrm{H}, \mathrm{d}$, aromatic), 7.68 $(1 \mathrm{H}, \mathrm{d}$, aromatic), $7.31(2 \mathrm{H}, \mathrm{dd}$, aromatic), $6.89(1 \mathrm{H}, \mathrm{d}$, aromatic $), 6.81(1 \mathrm{H}$, t, aromatic), $6.69\left(1 \mathrm{H}, \mathrm{d}\right.$, aromatic), $3.07(1 \mathrm{H}, \mathrm{m}, \mathrm{NH}), 2.19\left(6 \mathrm{H}, \mathrm{s}, \mathrm{CH}_{3}\right)$, ${ }^{13} \mathrm{C}-\mathrm{NMR}:(400 \mathrm{MHz})\left(\mathrm{CDCl}_{3}\right) \delta: 138.88,135.11,134.30,133.83,133.66$, $132.01,128.50,127.81,125.83,122.08,119.81,14.21,8.65$.

General procedure for the synthesis of allyl derivatives (6-11)

A solution of 2-bromo- $N$-methyl/2,3DMA-benzenesulfonamide $(0.8$ $\mathrm{mmol})$ in DMF $(5 \mathrm{ml})$ was added to the $\mathrm{n}$-hexane washed suspension of sodium hydride $(0.06 \mathrm{~g}$ of $60 \%, 1.6 \mathrm{mmol})$ in DMF $(2 \mathrm{ml})$. The solution was allowed to stir for half an hour at room temperature. Then un/substituted allylbromide $(0.99 \mathrm{mmol})$ was added to the above solution and allowed to stir at room temperature for 2-3 hrs. The progress of the reaction was observed by TLC. The mixture was poured on ice and $\mathrm{pH}$ was adjusted at 3-4. Precipitates obtained were further purified on column chromatography and dried over anhydrous $\mathrm{Na}_{2} \mathrm{SO}_{4}$ for spectral studies.

$\boldsymbol{N}$-Allyl-2-bromo- $\mathrm{N}$-methyl-benzenesulfonamide (6)

Light Yellow oil, Yield: $78 \%$, IR (neat) $v_{:}: 1329,1153 \mathrm{~cm}^{-1},{ }^{1} \mathrm{H}-\mathrm{NMR}$ : $(400 \mathrm{MHz})\left(\mathrm{CDCl}_{3}\right) \delta: 7.98(1 \mathrm{H}, \mathrm{d}$, aromatic $), 7.65(1 \mathrm{H}, \mathrm{d}$, aromatic $), 7.38$ $(2 \mathrm{H}, \mathrm{m}$, aromatic $), 5.76(1 \mathrm{H}, \mathrm{m}, \mathrm{CH}), 5.24\left(2 \mathrm{H}, \mathrm{dd},=\mathrm{CH}_{2}\right), 5.24\left(2 \mathrm{H}, \mathrm{d}, \mathrm{CH}_{2}\right)$, $2.68\left(3 \mathrm{H}, \mathrm{s}, \mathrm{CH}_{3}\right) .,{ }^{13} \mathrm{C}-\mathrm{NMR}:(400 \mathrm{MHz})\left(\mathrm{CDCl}_{3}\right) \delta: 143.26,139.86,139.05$ $136.97,135.78,133.93,123.43,122.90,54.57,35.88$ (7)

2-Bromo- $N$-methyl- $N$-(3-methylbut-2-en-1-yl)benzenesulfonamide

Light Yellow oil, Yield: $79.8 \%$, IR (neat) $v_{\text {max }}: 1332,1158 \mathrm{~cm}^{-1},{ }^{1} \mathrm{H}-\mathrm{NMR}$ : $(400 \mathrm{MHz})\left(\mathrm{CDCl}_{3}\right) \delta: \quad 8.17(1 \mathrm{H}, \mathrm{d}$, aromatic $), 7.72(1 \mathrm{H}, \mathrm{d}$, aromatic $), 7.42$ 
$\left(2 \mathrm{H}, \mathrm{dd}\right.$, aromatic), $5.18(1 \mathrm{H}, \mathrm{t}, \mathrm{CH}), 3.82\left(2 \mathrm{H}, \mathrm{d}, \mathrm{CH}_{2}\right), 2.79\left(3 \mathrm{H}, \mathrm{s}, \mathrm{NCH}_{3}\right)$ $1.61\left(3 \mathrm{H}, \mathrm{s}, \mathrm{CH}_{3}\right), 1.52\left(3 \mathrm{H}, \mathrm{s}, \mathrm{CH}_{3}\right),{ }^{13} \mathrm{C}-\mathrm{NMR}:(400 \mathrm{MHz}) \quad\left(\mathrm{CDCl}_{3}\right) \delta$ : $138.85,137.67,135.63,133.34,132.27,120.43,118.78,47.30,33.75,25.78$, 17.70

2-Bromo- $N$-methyl- $N$-(3-phenyl-allyl) benzenesulfonamide (8)

Pale Yellow oil, Yield: $67 \%$, IR (neat) $v_{\text {ax }}: 1331,1158 \mathrm{~cm}^{-1},{ }^{1} \mathrm{H}-\mathrm{NMR}$ : $(400 \mathrm{MHz})\left(\mathrm{CDCl}_{3}\right) \delta: 8.04-7.59\left(9 \mathrm{H}\right.$, aromatic), $6.43\left(1 \mathrm{H}, \mathrm{d},=\mathrm{CH}^{3} J=19.5\right)$, $5.98\left(1 \mathrm{H}, \mathrm{m},=\mathrm{CH}^{3} \mathrm{~J}=19.5 \mathrm{~Hz}\right), 3.94\left(2 \mathrm{H}, \mathrm{d}, \mathrm{CH}_{2}\right), 2.18\left(3 \mathrm{H}, \mathrm{s}, \mathrm{NCH}_{3}\right)$. ${ }^{13} \mathrm{C}-\mathrm{NMR}:(400 \mathrm{MHz})\left(\mathrm{CDCl}_{3}\right) \delta$ : $162.62,138.65,136.68,136.16,135.72$, $134.49,133.79,132.39,131.77,128.85,128.51,126.20,123.95,120.34,52.30$, 33.56 ,

$\mathrm{N}$-Allyl-2-bromo- $\mathrm{N}$-(2,3-dimethyl-phenyl)-benzenesulfonamide (9)

Brown solid, Yield: $78.3 \%$, MP: $112-113{ }^{\circ} \mathrm{C}$, IR $(\mathrm{KBr}) v_{\max }$ : 1334,1158 $\mathrm{cm}^{-1},{ }^{1} \mathrm{H}-\mathrm{NMR}:(400 \mathrm{MHz})\left(\mathrm{CDCl}_{3}\right) \delta: 7.68(2 \mathrm{H}, \mathrm{d}$, aromatic), $7.26(2 \mathrm{H}, \mathrm{m}$, aromatic), $7.01(1 \mathrm{H}, \mathrm{d}$, aromatic), $6.87(1 \mathrm{H}, \mathrm{t}$, aromatic), $6.63(1 \mathrm{H}, \mathrm{d}$, aromatic), $5.81(1 \mathrm{H}, \mathrm{m},=\mathrm{CH}), 4.94\left(2 \mathrm{H}, \mathrm{d},=\mathrm{CH}_{2}\right), 4.34\left(2 \mathrm{H}, \mathrm{d}, \mathrm{CH}_{2}\right) .{ }^{13} \mathrm{C}-\mathrm{NMR}:(400$ $\mathrm{MHz})\left(\mathrm{CDCl}_{3}\right) \delta: \quad 139.46,138.08,136.53,135.56,133.16,132.88,129.59$, $128.38,127.37,125.50,120.05,119.26,56.14,29.70,22.67,20.54,14.97$.

2-Bromo-N-(2,3-dimethyl-phenyl)-N-(3-methyl-but-2-enyl) benzene sulfonamide (10)

Brown viscous liquid, Yield: $98 \%$, IR (neat) $v_{\max }: 1341,1159 \mathrm{~cm}$ ', ${ }^{1} \mathrm{H}-\mathrm{NMR}:(400 \mathrm{MHz}) \quad\left(\mathrm{CDCl}_{3}\right) \delta: \quad 7.74(2 \mathrm{H}, \mathrm{max}$, aromatic $), 7.29$ $(2 \mathrm{H}, \mathrm{m}$, aromatic), $7.01(1 \mathrm{H}, \mathrm{d}$, aromatic), $6.89(1 \mathrm{H}, \mathrm{t}$, aromatic), $6.69(1 \mathrm{H}, \mathrm{d}$, aromatic), $5.21(1 \mathrm{H}, \mathrm{t},=\mathrm{CH}), 4.48\left(1 \mathrm{H}, \mathrm{q}, \mathrm{CH}_{2}\right), 4.31\left(1 \mathrm{H}, \mathrm{q}, \mathrm{CH}_{2}\right), 2.20(3 \mathrm{H}, \mathrm{s}$, $\left.\mathrm{CH}_{3}\right), 2.08\left(3 \mathrm{H}, \mathrm{s}, \mathrm{CH}_{3}\right), 1.58\left(3 \mathrm{H}, \mathrm{s},=\mathrm{CCH}_{3}\right), 1.32\left(3 \mathrm{H}, \mathrm{s},=\mathrm{CCH}_{3}\right) .{ }^{13} \mathrm{C}-\mathrm{NMR}$ : $(400 \mathrm{MHz})\left(\mathrm{CDCl}_{3}\right) \delta: 141.21,138.70,138.62,137.95,135.94,133.62,133.20$, $130.17,128.73,127.72,125.84,120.43,119.34,51.30,26.10,20.92,18.34$, $17.84,15.31$.

2-Bromo- $N$-(2,3-dimethyl-phenyl)- $N$-(3-phenyl-allyl)-benzene sulfonamide (11)

Brown viscous solid, Yield: $63 \%$, IR $(\mathrm{KBr}) v_{\mathrm{m}}: 1331,1158 \mathrm{~cm}^{-1}$, 'H-NMR: (400 MHz) $\left(\mathrm{CDCl}_{3}\right) \delta$ : 8.14-7.52 $(12 \mathrm{H}$, aromatic), $6.49(1 \mathrm{H}, \mathrm{d},=\mathrm{CH}$ $\left.{ }^{3} J=19.5\right), 5.79\left(1 \mathrm{H}, \mathrm{m},=\mathrm{CH}^{3} J=19.5 \mathrm{~Hz}\right), 4.02\left(2 \mathrm{H}, \mathrm{d}, \mathrm{CH}_{2}\right), 1.17(6 \mathrm{H}$, s, $\left.\mathrm{CH}_{3}\right) .{ }^{13} \mathrm{C}-\mathrm{NMR}:(400 \mathrm{MHz})\left(\mathrm{CDCl}_{3}\right) \delta: \quad 143.11,141.51,139.71,138.73$, $138.01,136.04,135.81,133.67,133.12,131.25,130.67,129.61,128.37$, $127.02,126.10,125.64,120.49,120.92,119.34,118.34,52.15,18.24,15.81$.

\section{Synthesis of cyclization products}

$N$-un/substituted Allyl-2-bromo- $N$-methyl/2,3-DMA-benzenesulfonamide $(0.482 \mathrm{mmol})$, catalytic amount of $\mathrm{Pd}(\mathrm{OAc})_{2}(0.0048 \mathrm{mmol})$ and $\mathrm{DIPA} / \mathrm{Et}_{3} \mathrm{~N}$ $(0.482 \mathrm{mmol})$ was added to the 1-methyl-2-pyrolidinone/toluene in round bottom flask $(25 \mathrm{ml})$ under $\mathrm{N}_{2}$. The mixture was subjected to heat for $24 \mathrm{hrs}$ at $100-120{ }^{\circ} \mathrm{C}$ with stirring. The different products were separated with prepHPLC technique using mixture of $20 \%$ AcOEt in n-hexane as mobile phase.

2,4-Dimethyl-2H-1,2-benzothiazine 1,1-dioxide (12)

Light Yellow oil, Yield: $38.2 \%$, IR (neat) $v_{\text {max }}: 1334,1176 \mathrm{~cm}^{-1},{ }^{1} \mathrm{H}-\mathrm{NMR}$ : $(400 \mathrm{MHz})\left(\mathrm{CDCl}_{3}\right) \delta: 7.82(1 \mathrm{H}, \mathrm{d}$, aromatic $), 7.59(1 \mathrm{H}, \mathrm{t}$, aromatic $), 7.42(2 \mathrm{H}$, $\mathrm{dd}$, aromatic), $6.25(1 \mathrm{H}, \mathrm{s}, \mathrm{CH}), 3.24\left(3 \mathrm{H}, \mathrm{s}, \mathrm{NCH}_{3}\right), 2.13\left(3 \mathrm{H}, \mathrm{s}, \mathrm{CH}_{3}\right)$. ${ }^{13} \mathrm{C}-\mathrm{NMR}:(400 \mathrm{MHz}),\left(\mathrm{CDCl}_{3}\right) \delta: \quad 134.20,132.06,131.05,129.79,127.35$, $124.50,121.59,113.57,34.40,16.21$,

2-Methyl-4-methylidene-3,4-dihydro-2 $\mathrm{H}$-1,2-benzothiazine 1,1-dioxide (13)

Light Yellow oil, Yield: $2.3 \%$, IR (neat) $v_{\max }: 1338,1179 \mathrm{~cm}^{-1},{ }^{1} \mathrm{H}-\mathrm{NMR}$ : $(400 \mathrm{MHz})\left(\mathrm{CDCl}_{3}\right) \delta: 7.92(1 \mathrm{H}, \mathrm{d}$, aromatic $), 7.44(2 \mathrm{H}, \mathrm{dd}$, aromatic), 7.33(1H, t, aromatic), $5.45\left(2 \mathrm{H}, \mathrm{s}, \mathrm{CH}_{2}\right), 4.03\left(2 \mathrm{H}, \mathrm{t}, \mathrm{CH}_{2}\right), 2.51\left(3 \mathrm{H}, \mathrm{s}, \mathrm{NCH}_{3}\right)$, ${ }^{13} \mathrm{C}-\mathrm{NMR}:(400 \mathrm{MHz})\left(\mathrm{CDCl}_{3}\right) \delta: \quad 147.69,139.16,133.98,133.09,131.12$, $129.87,129.19,99.35,49.29,34.56$

\section{2-Methyl-2,3-dihydro-1,2-benzothiazepine 1,1-dioxide (14)}

Light Yellow oil, Yield: $37.3 \%$, IR (neat) $v_{\text {an }}: 1341,1169 \mathrm{~cm}^{-1},{ }^{1} \mathrm{H}-\mathrm{NMR}$ : $(400 \mathrm{MHz})\left(\mathrm{CDCl}_{3}\right) \delta: 7.96(1 \mathrm{H}, \mathrm{d}$, aromatic $), 7.43(2 \mathrm{H}, \mathrm{dd}$, aromatic $), 7.29(1 \mathrm{H}$, $\mathrm{t}$, aromatic), $6.46(1 \mathrm{H}, \mathrm{d}, \mathrm{CH}), 5.82(1 \mathrm{H}, \mathrm{dd}, \mathrm{CH}), 4.23\left(2 \mathrm{H}, \mathrm{t}, \mathrm{CH}_{2}\right), 2.59(3 \mathrm{H}$, s, $\left.\mathrm{NCH}_{3}\right) \cdot{ }^{13} \mathrm{C}-\mathrm{NMR}:(400 \mathrm{MHz})\left(\mathrm{CDCl}_{3}\right) \delta: 138.67,133.76,132.96,130.28$, $129.45,128.90,127.43,121.49,52.90,35.46$,

2-(2,3-Dimethyl-phenyl)-4-methyl-2H-benzo[e][1,2] thiazine 1,1-dioxide (15)

Light Brown powder, Yield: 38.12\%, MP: $98-100{ }^{\circ} \mathrm{C}, \mathrm{IR}(\mathrm{KBr}) v_{\max }$ 1331, $1155 \mathrm{~cm}^{-1}$, ${ }^{\mathrm{H}} \mathrm{H}-\mathrm{NMR}$ : (400 MHz) $\left(\mathrm{CDCl}_{3}\right) \delta: 7.92(1 \mathrm{H}, \mathrm{d}$, aromatic), 7.61 $(1 \mathrm{H}, \mathrm{t}$, aromatic), $7.48(2 \mathrm{H}, \mathrm{dd}$, aromatic $), 7.28(1 \mathrm{H}, \mathrm{t}$, aromatic $), 7.12(2 \mathrm{H}$ $\mathrm{m}$, aromatic), $6.47(1 \mathrm{H}, \mathrm{s},=\mathrm{CH}), 2.23\left(3 \mathrm{H}, \mathrm{s}, \mathrm{CH}_{3}\right), 2.08\left(3 \mathrm{H}, \mathrm{s}, \mathrm{CH}_{3}\right), 1.49$ $(3 \mathrm{H}, \mathrm{s}, \mathrm{CH}) \cdot{ }^{13} \mathrm{C}-\mathrm{NMR}:(400 \mathrm{MHz})(\mathrm{CDCl}) \delta: 139.56,138.59,136.97,136.40$, $134.48,133.97,133.04,132.06,131.84,130.91,129.72,128.21,127.63,56.55$, $21.13,16.28,14.68$.

2-(2,3-Dimethylphenyl)-4-methylidene-3,4-dihydro-2H-1,2benzothiazine 1,1-dioxide (16)
Light brown powder, Yield: $5.6 \%$, MP: $115-116{ }^{\circ} \mathrm{C}$, IR (KBr) $v_{\max }: 1337$, $1155 \mathrm{~cm}^{-1},{ }^{1} \mathrm{H}-\mathrm{NMR}:(400 \mathrm{MHz})\left(\mathrm{CDCl}_{3}\right) \delta: 7.83(1 \mathrm{H}, \mathrm{d}$, aromatic), $7.59(1 \mathrm{H}$, t, aromatic), $7.42(2 \mathrm{H}, \mathrm{dd}$, aromatic), $7.22(1 \mathrm{H}, \mathrm{t}$, aromatic), $7.13(2 \mathrm{H}, \mathrm{m}$, aromatic), $5.16\left(2 \mathrm{H}, \mathrm{s}, \mathrm{CH}_{2}\right), 5.06\left(1 \mathrm{H}, \mathrm{d},=\mathrm{CH}_{2}\right), 4.24\left(1 \mathrm{H}, \mathrm{d},=\mathrm{CH}_{2}\right), 2.13(3 \mathrm{H}$, $\left.\mathrm{s}, \mathrm{CH}_{3}\right), 2.21\left(3 \mathrm{H}, \mathrm{s}, \mathrm{CH}_{3}\right), 2.12\left(3 \mathrm{H}, \mathrm{s}, \mathrm{CH}_{3}\right) \cdot{ }^{2} \mathrm{C}-\mathrm{NMR}:(400 \mathrm{MHz})\left(\mathrm{CDCl}_{3}\right)$ $\delta: 138.80,136.63,133.97,132.04,130.90,130.45,129.79,127.79,127.37$, $126.98,126.28,124.25,122.27,114.32,20.49,16.27,14.67$.

6-(2,3-Dimethyl-phenyl)-6,7-dihydro-5-thia-6-aza-benzocycloheptene 5,5-dioxide (17)

Light Brown solid, Yield: $33.41 \%$, MP: $87-88{ }^{\circ} \mathrm{C}$, IR $(\mathrm{KBr}) v_{\max }$ : 1339 , $1157 \mathrm{~cm}^{-1},{ }^{1} \mathrm{H}-\mathrm{NMR}:(400 \mathrm{MHz})\left(\mathrm{CDCl}_{3}\right) \delta: 7.87\left(1 \mathrm{H}, \mathrm{d}\right.$, aromatic) ${ }^{\max } 7.58(1 \mathrm{H}$, $\mathrm{t}$, aromatic), $7.51(2 \mathrm{H}, \mathrm{dd}$, aromatic), $6.93(1 \mathrm{H}, \mathrm{t}$, aromatic $), 6.54(1 \mathrm{H}, \mathrm{d},=\mathrm{CH}-$ ), $6.29(2 \mathrm{H}, \mathrm{dd}$, aromatic $), 6.09(1 \mathrm{H}, \mathrm{m},=\mathrm{CH}-), 4.21\left(2 \mathrm{H}, \mathrm{d}, \mathrm{CH}_{2}\right), 2.12(6 \mathrm{H}$, s, $\left.\mathrm{CH}_{3}\right) .{ }^{13} \mathrm{C}-\mathrm{NMR}:(400 \mathrm{MHz})\left(\mathrm{CDCl}_{3}\right) \delta: 138.81,136.63,134.79,133.54$, $132.26,130.09,129.18,128.85,127.38,126.42,125.15,123.78,114.42,55.05$, $20.68,14.97,14.45$.

2-Methyl-4-(propan-2-yl)-2H-1,2-benzothiazine 1,1-dioxide (18)

Light Yellow oil, Yield: $23.9 \%$, IR (neat) $v_{\max }$ : 1334, $1156 \mathrm{~cm}^{-}$ , ${ }^{1} \mathrm{H}-\mathrm{NMR}:(400 \mathrm{MHz})\left(\mathrm{CDCl}_{3}\right) \delta: 7.81(1 \mathrm{H}, \mathrm{d}$, aromatic), $7.55(2 \mathrm{H}, \mathrm{dd}$, aromatic), $7.48(1 \mathrm{H}, \mathrm{m}$, aromatic $), 6.21(1 \mathrm{H}, \mathrm{s}, \mathrm{CH}), 3.24\left(3 \mathrm{H}, \mathrm{s}, \mathrm{NCH}_{3}\right), 3.06$ $(1 \mathrm{H}, \mathrm{m}, \mathrm{CH}), 1.17\left(6 \mathrm{H}, \mathrm{s}, \mathrm{CH}_{3}\right) .{ }^{13} \mathrm{C}-\mathrm{NMR}:(400 \mathrm{MHz})\left(\mathrm{CDCl}_{3}\right) \delta: \quad 132.26$, $131.82,129.34,127.56,125.40,123.67,122.71,117.50,34.51,27.05,22.14$,

2,5,5-Trimethyl-2,5-dihydro-1,2-benzothiazepine 1,1-dioxide (19)

Light Yellow oil, Yield: $23.9 \%$, IR (neat) $v_{\max }: 1328,1164 \mathrm{~cm}^{-1},{ }^{1} \mathrm{H}-\mathrm{NMR}$ : $(400 \mathrm{MHz})\left(\mathrm{CDCl}_{3}\right) \delta: 7.76-7.41(4 \mathrm{H}$, aromatic), $4.23(1 \mathrm{H}, \mathrm{d}, \mathrm{CH}), 4.98(1 \mathrm{H}, \mathrm{d}$, $\mathrm{CH}), 2.51\left(3 \mathrm{H}, \mathrm{s}, \mathrm{NCH}_{3}\right), 1.48\left(6 \mathrm{H}, \mathrm{s}, \mathrm{CH}_{3}\right) .,{ }^{13} \mathrm{C}-\mathrm{NMR}:(400 \mathrm{MHz})\left(\mathrm{CDCl}_{3}\right) \delta$ : $134.62,133.62,132.15,131.53,127.78,124.23,122.46,119.65,52.25,41.76$, 35.26 ,

2-(2,3-Dimethyl-phenyl)-4-(propan-2-yl)-2H-1,2-benzothiazine 1,1-dioxide (20)

Light brown paste, Yield: $29.46 \%$, IR (neat) $v_{\max }: 1332,1158 \mathrm{~cm}$ 1, ${ }^{1} \mathrm{H}$-NMR: (400 MHz) $\left(\mathrm{CDCl}^{2}\right) \delta: \quad 7.81(1 \mathrm{H}, \mathrm{d}$, aromatic), $7.55(2 \mathrm{H}, \mathrm{dd}$, aromatic), $7.48\left(1 \mathrm{H}, \mathrm{m}\right.$, aromatic), $6.21(1 \mathrm{H}, \mathrm{s}, \mathrm{CH}), 3.24\left(3 \mathrm{H}, \mathrm{s}, \mathrm{NCH}_{3}\right), 3.06$ $(1 \mathrm{H}, \mathrm{m}, \mathrm{CH}), 1.17\left(6 \mathrm{H}, \mathrm{s}, \mathrm{CH}_{3}\right) .{ }^{13} \mathrm{C}-\mathrm{NMR}:(400 \mathrm{MHz})\left(\mathrm{CDCl}_{3}\right) \delta: \quad 132.26$, $131.82,129.34,127.56,125.40,123.67,122.71,117.50,34.51,27.05,22.14$

6-(2,3-Dimethyl-phenyl)-9,9-dimethyl-6,9-dihydro-5-thia-6-azabenzocycloheptene 5,5-dioxide (21)

Light brown paste, Yield: $26.35 \%$, IR (neat) $v_{\text {an }}: 1342,1168 \mathrm{~cm}^{-1}$, 'H-NMR: (400 MHz) $\left(\mathrm{CDCl}_{3}\right) \delta: 7.76-7.41(4 \mathrm{H}, \mathrm{m}$, aromatic), $4.23(1 \mathrm{H}, \mathrm{d}$, $\mathrm{CH}), 4.98(1 \mathrm{H}, \mathrm{d}, \mathrm{CH}), 2.51\left(3 \mathrm{H}, \mathrm{s}, \mathrm{NCH}_{3}\right), 1.48\left(6 \mathrm{H}, \mathrm{s}, \mathrm{CH}_{3}\right) \cdot{ }^{13} \mathrm{C}-\mathrm{NMR}:(400$ MHz) $\left(\mathrm{CDCl}_{3}\right) \delta: 134.62,133.62,132.15,131.53,127.78,124.23,122.46$, $119.65,52.25,41.76,35.26$,

4-Benzylidene-2-methyl-3,4-dihydro- $2 \mathrm{H}$-benzo $[e][1,2]$ thiazine 1,1-dioxide (22)

Pale Yellow oil, Yield: $54 \%$, IR (neat) $v_{\text {ax }}: 1325,1157 \mathrm{~cm}^{-1},{ }^{1} \mathrm{H}-\mathrm{NMR}$ : $(400 \mathrm{MHz})\left(\mathrm{CDCl}_{3}\right) \delta: 7.86-7.43(9 \mathrm{H}, \operatorname{aromatic}), 6.23(1 \mathrm{H}, \mathrm{s},=\mathrm{CH}), 3.83(2 \mathrm{H}$, s, $\left.\mathrm{CH}_{2}\right), 3.24\left(3 \mathrm{H}, \mathrm{s}, \mathrm{CH}_{3}\right) .,{ }^{13} \mathrm{C}-\mathrm{NMR}:(400 \mathrm{MHz})\left(\mathrm{CDCl}_{3}\right) \delta: 132.51,132.00$, $131.83,131.66,129.28,128.96,128.44,128.31,127.21,126.62,125.47$, $124.70,124.42,122.04,51.92,34.13$

4-Benzylidene-2-(2,3-dimethyl-phenyl)-3,4-dihydro-2 $H$-benzo $[e][1,2]$ thiazine 1,1-dioxide (23)

Brown powder, Yield: $54 \%$, MP: $202-204{ }^{\circ} \mathrm{C}$, IR (KBr) $v_{\max }: 1329,1154$ $\mathrm{cm}^{-1},{ }^{1} \mathrm{H}-\mathrm{NMR}:(400 \mathrm{MHz})\left(\mathrm{CDCl}_{3}\right) \delta: \quad 8.02-7.21(12 \mathrm{H}$, aromatic $), 6.08(1 \mathrm{H}$, $\mathrm{s}$, =CH), $4.11\left(2 \mathrm{H}, \mathrm{s}, \mathrm{CH}_{2}\right), 2.97\left(6 \mathrm{H}, \mathrm{s}, \mathrm{CH}_{3}\right) \cdot{ }^{13} \mathrm{C}-\mathrm{NMR}:(400 \mathrm{MHz})\left(\mathrm{CDCl}_{3}\right)$ $\delta: 149.05,147.24,142.17,139.03,138.26,137.41,135.18,134.76,133.52$, $131.68,130.18,129.29,128.01,127.31,126.09,125.85,121.51,120.06$, $119.11,117.34,53.51,19.91,16.57$.

\section{RESULTS AND DISCUSSIONS}

The present work covers the development of new research methodology headed for the synthesis of sultams. The cheap and readily available $o$-aminobenzenesulfonic acid (orthanilic acid) (1) was used as starting material (Scheme 1). Diazotization of (1) led to $o$-bromobenzenesulfonic acid which was treated with base $\mathrm{NaOH}$ to produce sodium $o$-bromobenzenesulfonate (2). The sulfonyl chloride (3) was isolated (50-60\%) following chlorination of (2) with $\mathrm{PCl}_{5}$ in a neat fashion ${ }^{44}$. The sulfonamide derivatives $(4,5)$ were synthesized in encouraging yields by direct reaction of sulfonyl chloride (3) with methyl amine hydrochloride and 2,3-dimethylaniline (2,3-DMA), respectively. $N$-alkylation of the sulfonamides $(\mathbf{4}, \mathbf{5})$ was carried out with three allyl bromide reagents i.e. allylbromide, 3,3-dimthylallylbromide and transcinnamyl bromide to get the corresponding derivatives (6-11). The compounds were characterized by spectroscopic techniques and used in the next scheme without further purification. 


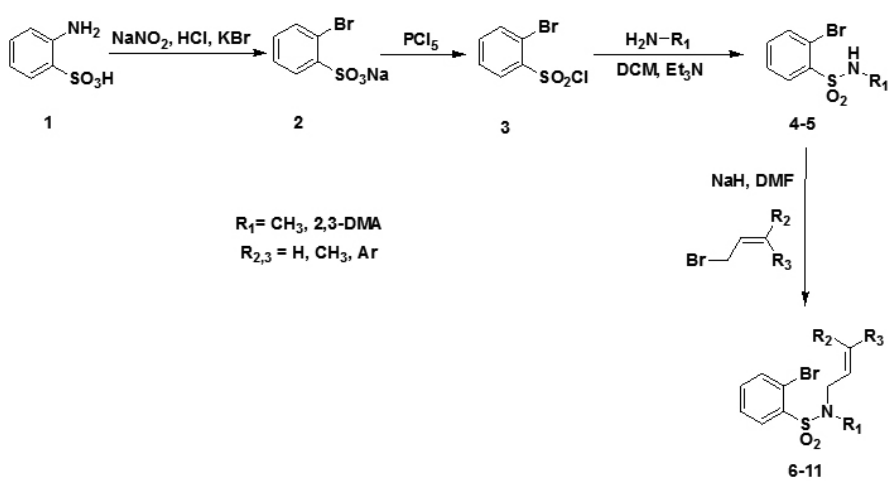

Scheme 1: Synthesis of sulfonamides

The six allyl-containing benzenesulfonamides (6-11) were subjected to $\mathrm{C}-\mathrm{C}$ cross-coupling by applying $\mathrm{Pd}(\mathrm{II})$ acetate under basic conditions (using di-isopropylamine) in either 1-methyl-2-pyrrolidinone or toluene as reaction medium. The attempt of cyclization was made without employing triphenylphosphine ligand which is mandatory for Heck cyclization. The pace of reaction was monitored by TLC. For first two reactions pyrrolidinone, while for rest of the four reactions toluene was employed as reaction medium for the sake of convenience in work-up. We isolated two isomeric analogues of benzothiazine along with benzothiazepine in diverse yields during first two cyclization attempts. The two analogues of benzothiazine differ in the position of double bond. The analogue in which double bond lies in the thiazine ring
(12) dominated in yield over the other exocyclic double bond containing isomer (13) $(38.2 \%$ and $2.3 \%$, respectively). The benzothiazepines $(\mathbf{1 4}, \mathbf{1 7})$ were also isolated in comparative yields $(37.3 \%, 33.4 \%)$. These isomeric products are the logical outcome of Heck coupling ${ }^{45,46}$ recipe.

The key feature in current research was not only to avoid usage of a bulky ligand, but also to derive the environment where only one required type of product could be the destination. Therefore, we carried out ligandfree palladium catalyzed cyclization to yield six-membered sultams, taking following reasons into consideration.

I. A good leaving group like iodo- instead of bromo- might facilitate the formation of six membered sultams.

II. Presence of a sterically crowded group at alkene terminal of the sulfonamides (6-11) might be supportive towards formation of one type of product only.

Many citation $\mathrm{s}^{47}$ have been found, which consider the first point as mere option to render significant outcomes. We jumped into the second option, used 3,3-dimethyl allyl and cinnamyl alkyl groups $(\mathbf{7}, \mathbf{8}, \mathbf{1 0}, \mathbf{1 1})$ to target the planned six membered cyclic molecules. 3,3-Dimethyl allyl group containing derivatives $(\mathbf{7}, \mathbf{1 0})$ yielded both six- $(\mathbf{1 8}, \mathbf{1 9})$ and seven- $(\mathbf{2 0}, \mathbf{2 1})$ membered sultams. Eventually, the cinnamyl sulfonamide derivatives $(\mathbf{8}, \mathbf{1 1})$ produced purely thiazine-containing cyclic products $(\mathbf{2 2}, \mathbf{2 3})$ with exo-cyclic double bond $(54 \%)$.

The overall results justify that the presence of bulky group at ' $\mathrm{N}$ ' of the sulfonamide $(\mathbf{4}, \mathbf{5})$, away from the point of arylation, does not contribute towards selection for six-/seven-membered products. Secondly, the crowding produced at point of $\mathrm{C}-\mathrm{C}$ cross-coupling $(\mathbf{8}, \mathbf{1 1})$ has exclusively decided the fate of final products in term of six-membered sultams $(\mathbf{2 2 , 2 3 )}$ only. This inconclusion is the success of present methodology.

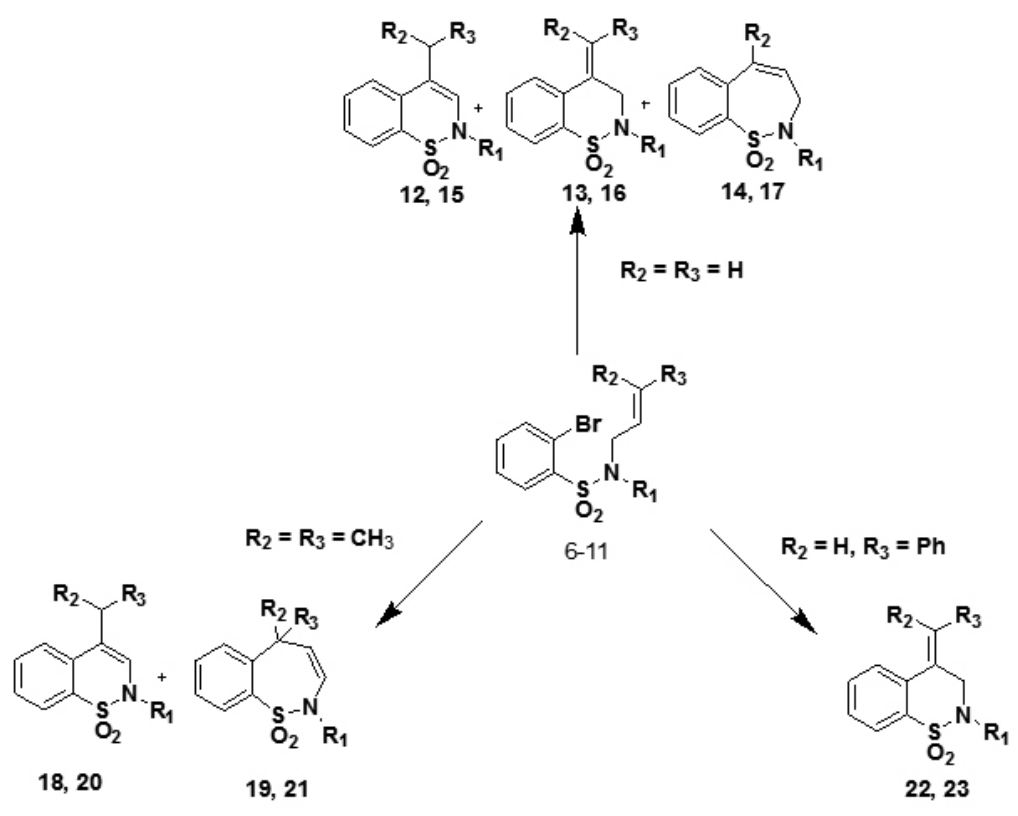

\begin{tabular}{|c|c|c|c|c|c|c|c|c|c|c|c|c|c|c|}
\hline No. & 4 & 5 & 6 & 7 & 8 & 9 & 10 & 11 & $12-14$ & $15-17$ & $18-19$ & $20-21$ & 22 & 23 \\
\hline $\mathrm{R} 1$ & $\mathrm{CH}_{3}$ & $\begin{array}{c}2, \\
\text { 3-DMA }\end{array}$ & $\mathrm{CH}_{3}$ & $\mathrm{CH}_{3}$ & $\mathrm{CH}_{3}$ & $\begin{array}{c}2, \\
\text { 3-DMA }\end{array}$ & $\begin{array}{c}2, \\
\text { 3-DMA }\end{array}$ & $\begin{array}{c}2, \\
\text { 3-DMA }\end{array}$ & $\begin{array}{c}\mathrm{CH}_{3} \\
\text { 3-DMA }\end{array}$ & $\begin{array}{c}\mathrm{CH}_{3} \\
\text { 2, 3-DMA }\end{array}$ & $\mathrm{CH}_{3}$ & $\begin{array}{c}2, \\
\text { 3-DMA }\end{array}$ \\
\hline $\mathrm{R} 2$ & - & - & $\mathrm{H}$ & $\mathrm{CH}_{3}$ & $\mathrm{H}$ & $\mathrm{H}$ & $\mathrm{CH}_{3}$ & $\mathrm{H}$ & $\mathrm{H}$ & $\mathrm{H}$ & $\mathrm{CH}_{3}$ & $\mathrm{CH}_{3}$ & $\mathrm{H}$ & $\mathrm{H}$ \\
\hline
\end{tabular}

Scheme 2: Synthesis of sultams 


\section{CONCLUSION}

The current studies explain the synthesis of six membered cyclic sultams in successive manner using Pd-catalysed chemistry without any ligand. The effects of simple and satirically hindered groups were evaluated in synthesis of targeted compounds to avoid isomeric products.

\section{ACKNOWLEDGEMENT}

The authors MNA (PIN 042-120607-Ps2-183) and WAS (pin 042-120639Ps158) acknowledge the support of Higher Education Commission (HEC), Islamabad, Pakistan.

\section{REFERENCES}

1. G. Ronald, Y. Mark, Tetr. Lett. 7255, 41, (2000)

2. A. Leo, R. D. Paquette, N. F. Dura, S. Marshall, J. Org. Chem. 8438, 71, (2006)

3. E. Paul, M. Thomas, S. M. Ben, R. Sophie, Org. Lett. 43, 7, (2005)

4. G. Ronald, S. Vladimir, T. Vasuki, Tetr. Lett. 3003, 41, (2000)

5. J. K. Stille, Angew. Chem., Int. Ed. Eng. 508, 25, (1986, 25, 508.

6. E. Negishi, Q. Hu, Z. Huang, M. Qian, G. Wang, Aldrichimica Acta. 71, $38,(2005)$

7. N. Miyaura, A. Suzuki, Chem Rev. 2457, 95, (1995)

8. T. Hiyama, J. Organomet. Chem. 58, 653, (2002)

9. R. F. Heck, Acc. Chem. Res. 146, 12, (1979)

10. W. A. Herrmann, C. P. Reisinger, M. Spiegler J. Organomet. Chem. 93, $557,(1998)$

11. F. Rataboul, A. Zapf, R. Jackstell, S. Harkal, T. Riermeier, A. Monsees, U. Dingerdissen, M. Beller, Chem. Eur. J. 2983, 10, (2004)

12. W. S. Knowles, Angew. Chem., Int . Ed. Eng. 1998, 41, (2002)

13. P. Catsoulacos, C. Camoutsis, J. Heterocycl. Chem. 1503, 16, (19790

14. R. R. Gupta, R. Kumar, J. Fluorine Chem. 19, 31, (1986)

15. V. Gupta, R. R. Gupta, J. Prakt. Chem. 153, 333, (1991)

16. R. R. Gupta, R. Kumar, R. K. Gutam, J. Fluorine Chem. 381, 28, (1985)

17. R. Wammack, M. Remzi, C. Seitz, B. Djavan, M. Marberger, Eur. Urol. $596,41,(2002)$

18. M. R. Pijak, P. Turcani, Z. Turcaniova, I. Buran, I. Gogolak, A. Mihal, F. Gazdik, Bratisl. Med. J. 469, 103, (2002)

19. K. Malagu, J. Boustie, M. David, J. Sauleau, M. Amoros,R. L. Girre, A Sauleau, Pharm. Pharmacol. Commun. 57, 4, (1998)

20. G. Trapani, A. Reho, F. Morlacchi, A. Latrofa, P. Marchini, F. Venturi, F. Cantalamessa, Farmaco. Ed. Sci. 369, 40, (1985)

21. M. Yaltirik, C. K. Oral, I. Oral, G. Kasaboglu, V. Cebi, Turk. J. Med. Sci. $151,31,(2001)$

22. M. S. Park, E. S. Chang, M. S. Lee, S. K. Kwon, Bull. Korean Chem. Soc. $1836,23,(2002)$

23. G. Laban, W. Guenther, D. Lohmann, Ger. Patent DD, 247674, (1987)

24. Alfred, B. Medicinal Chemistry, 2nd eds. Interscience, NY. (London) 1960.

25. C. A. Bretting and S. G. Grue, Brit. Patents GB, 2,207130, 34, (1989)

26. M. Zia-ur-Rehman, J. A. Choudary, M. R. J. Elsegood, H. L. Siddiqui, K. M. Khan, Eur. J. Med. Chem., 1311, 44, (2009)

27. I. Takemoto, K. Yamasaki, H. Kaminaka, Biosci, Biotechnol. Biochem. $788,58,(1994)$

28. G. P. J. Schroter, M. Hoelscher, C. W. Putman, K. A. Porter, T. E. Starzl, Ann. Surg. 115, 186, (1977)

29. V. Cecchetti, V. Caldrone, O. Tabarrini, S. Sabatini, E. Filipponi, L. Testai, R. Spogli, E. Martinotti, A. Fravolini, J. Med. Chem. 3670, 46, (2003)

30. J. V. Braun, Chem. Ber. 2332, 56, (1923)

31. J. G. Lombardino, Wiseman E. J. Med. Chem. 848, 15, (1972)

32. M. Harmata, X. Hong, J. Am. Chem. Soc., 5754, 125, (2003)

33. M. Harmata, X. Hong, S. K. Ghosh, Tetr. Lett. 5233, 45, (2004)

34. K. Abe, S. Yamamoto, K. Matsui, J. Pharm. Soc. Jp., 1058, 76, (1956)

35. V. Anil, T. Pei-San, W. D. Stevan Tetr. Lett. 8591, 47, (2006)

36. D. K. Rayabarapu, A. Zhou, K. O. Jeon, T. Samarakoon, A. Rolf, H. Siddiqui, P. R. Hanson, Tetrahed. 3180, 65, (2009)

37. W. A. Siddiqui, S. Ahmad, I. U. Khan, H. L. Siddiqui, V. U. Ahmad, J. Chem. Soc. Pak. 44, 29, (2007)

38. W. A. Siddiqui, S. Ahmad, I. U. Khan, H. L. Siddiqui, G. W. Weaver, Synth. Comm., 767, 37, (2007)

39. W. A. Siddiqui, S. Ahmad, H. L. Siddiqui, M. A. Tariq, M. Parvez, Acta
Cryst. E. o4585, 63, (2007)

40. M. N. Arshad, M. N. Tahir, I. U. Khan, M. Shafiq, W. A. Siddiqui, Acta Cryst. E. o2045, 64, (2008)

41. M. Zia-ur-Rehman, J. A .Choudary, M. R. J. Elsegood, H. L. Siddiqui, K. M. Khan, Eur. J. Med. Chem. 1311, 44, (2009)

42. M. N. Arshad, O. ahin, M. Zia-ur-Rehman, M. shafiq, I. U. Khan, A. M. Asiri, S. B. Khan, K. A. Alamry, J Chem. Cryst. 671, 43, (2013)

43. M. N. Arshad, O. ${ }^{a}$ ahin, M. Zia-ur-Rehman, I. U. Khan, A. M. Asiri, H. M. Rafique, J. Struct. Chem. 437, 54, (2013)

44. M. M. Chau, J. L. Kice, J. Org. Chem. 3265, 42, (1977)

45. . Koerber-Plé, G. Massiott, Synlett. 759, (1994)

46. S. E. Gibson, N. Guillo, R. J. Middleton, A. Thuillez, M. J. Tozer, J. Chem. Soc. Perkin Trans. 1, 447, (1997)

47. C. Amatore, M. Azzabi, A. Jutand, J. Am. Chem. Soc. 8375, 113, (1991) 